\title{
Platooning With DSRC-Based IVC-Enabled Autonomous Vehicles: Adding Infrared Communications for IVC Reliability Improvement
}

\author{
Pedro Fernandes, Member, IEEE, and Urbano Nunes, Senior Member, IEEE
}

\begin{abstract}
Platooning with IVC-enabled autonomous vehicles may enable a significant increase in lane capacity, if performed with constant spacing policies. However, to be effective, such system is very demanding with respect to communication performance and reliability. Dedicated short range communications (DSRC) is the prominent intervehicle communication (IVC) technology. However, its reliability rises concerns when operating under platooning scenarios. In this paper we identify some specific problems that platooning pose to DSRC, through the simulation of several scenarios implemented in the NS-3 network simulator. Moreover, we propose a new concept of IVC when applied to platoons, using simultaneously two different communication technologies: DSRC and infrared (IR). New guidelines toward more efficient use of IVC transmission media are suggested, e.g., by broadcasting the event-driven type of messages through DSRC, whereas periodic vehicle control-based messages use the IR channel, in unicast. Furthermore, the base architecture of the IVC proposed system is presented.
\end{abstract}

\section{INTRODUCTION}

Urban traffic congestion is a major problem of modern societies. The associated costs are beyond simple computations and would demand a vast long-term research by itself. Intelligent Transportation Systems (ITS) may contribute to alleviate this problem. Among the myriad of concepts it encompasses, intervehicle communications (IVC) in all its forms, e.g., vehicle-to-vehicle (V2V), or vehicle-toinfrastructure (V2I) commonly referred as V2X, consist of a set of technological resources that can foster new approaches enabling ITS on urban mobility improvement.

Platooning may help to improve lane capacity, if constant spacing is used [1]. Additionally, some conditions should be met to ensure both high traffic flow and traffic density: the use of IVC-enabled autonomous vehicles; autonomous vehicles do not share the road with conventional vehicles; autonomous vehicles use dedicated tracks, and operate on a nonstop basis from origin to destination [2]; tracks have off-line stations; vehicles' maneuvers exiting the tracks to stations, as well as entering the tracks from stations, are performed cooperatively; the system is managed by a hierarchical advanced traffic management system (ATMS).

IVC have been rapidly evolving. For instance, Dedicated Short Range Communications/Wireless Access in Vehicular Environments (DSRC/WAVE) [3], [4], [5] has been showing its high potential on enabling ITS related technologies, such as autonomous vehicles [6], adaptive crui-

This work was supported by Portuguese Foundation for Science and Technology under Grant PTDC/SEN-TRA/099413/2008.

The authors are with the Institute of Systems and Robotics, Department of Electrical and Computer Engineering, University of Coimbra, 3030-290 Coimbra, Portugal (e-mail: pedro@isr.uc.pt; urbano@isr.uc.pt). se control (ACC) [7], cooperative adaptive cruise control (CACC) [8], [9], cooperative active safety system (CASS) [10], on-ramp merging systems [11], [12], among others. Long term evolution advanced (LTE-Advanced) [13] is another promising communication standard, finally allowing to envisage broadband communication systems to be widely used on ITS.

IVC are conceived for three main applications [14]: information and warning functions, communication-based longitudinal control and cooperative assistance systems. Concerning the message types, two distinct groups are considered [15]: event-driven and periodic. For each message type, different strategies are implemented: event-driven (e.g., reporting a traffic accident) are sent with high priority; periodic messages (e.g., reporting the status of a vehicle) require to be sent at a frequency of ten messages per second. When used under scenarios comprising high IVCenabled vehicle density, this strategy presents the drawback of causing channel congestion. Remarkably, the platooning scenarios considered in this paper may be characterized under all the three types of main applications (information and warning functions, communication-based longitudinal control and cooperative assistance systems), and use both message types (event-driven and periodic). Therefore, DSRC is pushed to the limit if used under such scenarios, and has to deal with contradictory constraints under stringent conditions, compromising DSRC reliability, as reported in [1]. However, other IVC technologies may come to DSRC rescue. Among them, ultra wideband (UWB) communications and infrared (IR) communications, are two promising IVC technologies that may help to unburden DSRC tasks. The former, operating around $60 \mathrm{GHz}$, have been more envisaged for intravehicular applications, due to its high bandwidth and very short range. The later, operating on a line-of-sight (LOS) basis, has been used mainly for tolling applications, albeit its enormous potential with respect to high bandwidth and security. However, both of them present some limitations, e.g., very short range, and sensitivity to adverse weather conditions. Nevertheless, their use should be considered for the very stringent IVC environments, such as platooning of autonomous vehicles using constant spacing policies. Their intrinsic features make them very strong candidates to be used in platoons, if mitigation of communication delays using anticipatory information, as proposed in [1], is used.

Of the aforementioned two IVC technologies, we consider IR as more appropriate to use in platooning environments, since it presents no interference whatsoever with DSRC. Moreover, since it is envisaged to be used by vehicles 
in platoon formation, LOS is not an unfavorable feature, but a desired one. Additionally, IR enables high privacy, does not need licensing, and bandwidth allocations are not required [16]. And a psychological factor may also be in favor of IR use: although there is no evidence of millimeter waves being potentially harmful to human health, the mere fact that many people may be afraid of that possibility, may lead them to avoid further electromagnetic waves exposure.

Communications Access for Land Mobiles (CALM) [17], [18] (formerly Continuous Air-interface Long and Medium range [19]) standard, presents a comprehensive framework to ensure interoperability among diverse communication systems. DSRC is referred as CALM-M5 [20], UWB roughly as CALM-MM [21], and IR as CALM-IR [22], just to mention a few. The International Organization for Standardization (ISO) [23] under the technical committee TC 204, and the European Telecommunications Standards Institute (ETSI) [24] under the TC ITS, are two of the standardization bodies actively developing CALM [25]. IR is used in several ITS projects, such as in the German Toll Collect system [26], in Malaysia, Korea, Taiwan and India in electronic toll collection systems, and in the Vehicle Information and Communication Systems (VICS) [27] in Japan.

In this paper we continue the analysis and simulation of DSRC protocols reported in [1], presenting further simulation scenarios in the NS-3 network simulator [28], and identifying some issues that broadcasted DSRC messages face with respect to reliability and safety. Subsequently, we propose some guidelines on the simultaneous use of two IVC technologies, complementing DSRC with the use of IR, to enable the improvement of IVC reliability and the safe operation of a constant spacing platooning system.

\section{RELATED WORK}

There are a vast number of published research works on IVC. For IVC surveys, see e.g., [14], [15], [29], [30], [31]. Safety-based IVC are crucial for appropriate constant spacing platooning operation. Although DSRC has been the prominent IVC technology in ITS context, DSRC broadcasting performance may rise some concerns with respect to reliability, for safe vehicle operation in constant spacing platooning scenarios [1]. Several other proposals have recently been made, aiming to improve DSRC characteristics. In this context, Yu and Biswas [32] presented a novel medium access control (MAC) protocol for IVC using DSRC, which consisted in a self-configuring time division multiple access protocol with short and deterministic delay bound capabilities. Torrent-Moreno et al. [33] proposed a distributed transmit power control method to control the load of periodic messages on the channel. Bi et al. [34] presented a crosslayer broadcast protocol to allow efficient and reliable message dissemination in IVC systems. Palazzi et al. [35] proposed a novel IVC architecture that adapts its functionalities to efficiently serve applications by quickly propagating their messages over a vehicular network. Tabatabaei et al. [36] presented an improvement on simpler propagation models for simulations by augmenting ray-tracing-derived models of wireless propagation. To our best knowledge there are no published research papers concerning the analysis of DSRC use in a constant spacing platooning environment, presenting specific problems to be dealt with.

The more appropriate mean to test IVC is through network simulators coupled to traffic simulators for realistic trace generation. Piorkowski et al. [37] proposed TraNS, a vehicular network simulator that integrates the Simulation of Urban MObility (SUMO) traffic simulator [38] and the NS-2 network simulator [39]. Wegener et al. [40] proposed TraCI, coupling the SUMO with other applications through a TCP-based client-server architecture. Simulators focused on Personal Rapid Transit (PRT) systems have also been proposed [41], [42]. However, until now, none of these simulators model constant spacing platooning of IVC-enabled autonomous vehicles.

In [43] we addressed the implementation of IVC-enabled autonomous vehicles platooning capabilities in the SUMO traffic simulator. Since platooning with a constant spacing policy is very sensitive to communication delays [44], in [1] we managed to improve platoon string stability using anticipatory information, both from the platoon's leader and the followers, with the use of the new proposed algorithms to mitigate communication delays on platoons of IVC-enabled autonomous vehicles. More recently, we used the modified SUMO simulator to validate traffic flow assumptions, as described in [1], and we implemented new scenarios to assess the feasibility of a complete multi-platooning system of IVC-enabled autonomous vehicles with off-line stations, performing cooperatively. In [45], [46], we proposed the base rules for interplatoon positioning management strategies, by maintaining a constant spacing between platoons' leaders, for safe and efficient ATMS operation, to ensure high traffic capacity and vehicle density, while avoiding traffic congestion. To our best knowledge, there are still no traffic simulators with the new presented features in [46], such as: the simulation of a large number of platoons of constant spaced autonomous vehicles; vehicles exiting to stations and entering the main track from stations cooperatively; the permanent measuring of the traffic flow, capacity, occupancy and vehicle density; and the avoidance of congestion operating at very high flow and density values.

Concerning the use of IR in IVC, Varaiya [47] already included IR as one possible IVC technology, back in 1993. In [48], Mio et al. presented experiments with an IR-LED for V2V. In [49], Fujii et al. presented studies on IVC with IR. More recently, Tsugawa [50] presented a comprehensive survey of IVC technologies, including IR communications. The European projects CO-OPerative SystEms for Intelligent Road Safety (COOPERS) [51] and Cooperative VehicleInfrastructure Systems [52], among others, have significantly fostered CALM development.

However, the main problem remains, and to our best knowledge, there are no published studies concerning platooning of IVC-enabled autonomous vehicles, using constant spacing policies and reliable communications, particularly with the use of more than one communication technology. 
TABLE I

IR AND DSRC CHARACTERISTICS AND FEATURES

\begin{tabular}{lll}
\hline \hline Type & IR & DSRC \\
\hline Communication mode & V2I \& V2V & V2I \& V2V \\
Directionality & One-/Two-way & One-/Two-way \\
Latency & Very low & $\leq 50 \mathrm{~ms}$ \\
Data rate & 1 to $128 \mathrm{Mb} / \mathrm{s}$ & 3 to $27 \mathrm{Mb} / \mathrm{s}$ \\
Range & until $10 \mathrm{~m}$ to $100 \mathrm{~m}$ & until $300 \mathrm{~m}$ to $1 \mathrm{~km}$ \\
& Bidirectional & Bidirectional \\
Transmission mode & and Broadcast & and Broadcast \\
Mobile station speed & up to $260 \mathrm{~km} / \mathrm{h}$ & up to $260 \mathrm{~km} / \mathrm{h}$ \\
Freq. band/Wavelength & $870 \mathrm{~nm}$ & $5.9 \mathrm{GHz}$ \\
\hline
\end{tabular}

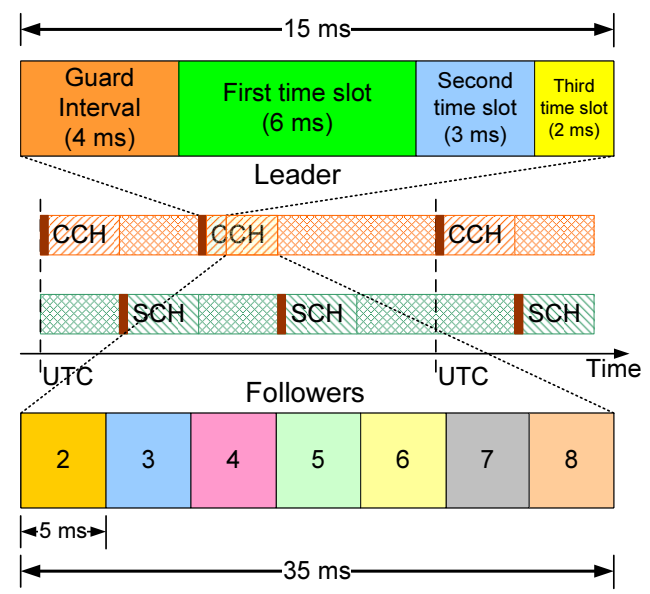

Fig. 1. DSRC CCH leaders' and followers' time slots (from [1]).

\section{INFRARED COMMUNICATIONS}

IR communications present very specific and interesting characteristics. Table I (adapted from [53] and [54]), presents some of the IR and the DSRC characteristics and features. Moreover, due to the directionality of IR beams, this communication technology is very appropriate to use on a single-lane basis, which is the case of the proposed platooning system. Additionally, other advantages of IR may be mentioned [53]: enables direct communication; provides fast link setup $(<10 \mathrm{~ms})$; has excellent focusing and beamshaping capabilities; supports multiple links within a communication zone; offers very high bandwidth; presents latencies and delays of few milliseconds; provides interference-free communications; and has a license-free wireless spectrum.

\section{NETWORK SIMULATION PARAMETERS}

Some of the information updating schemes used in [1] are very demanding in what concerns communication network load. And the current IVC technologies and protocols rise some concerns of whether they can cope with the involved timings of the information updating schemes, when all vehicles communicate within $100 \mathrm{~ms}$. So we chose to test DSRC operation in such scenarios, using the NS-3 network simulator. The DSRC system parameters are the same as presented in Table VIII in [1], and also described in [1], in the Section VIII, Subsection A.
To assess proper communication flow, a 10-platoon setup of eight vehicles each, was implemented. The main NS-3 simulation parameters are the same as in Table IX of [1].

\section{PLATOONING SCENARIOS USING DSRC}

The first platooning scenario, using a single lane platooning, is similar to that reported in [1], Section VIII, Subsection B. The second one, using two single lanes platooning, consists of an elevated crossroad, with two perpendicular tracks $4 m$ vertically apart.

\section{A. NS-3 Simulation Setup of a Single Lane Platooning}

A scenario of ten platoons of eight-vehicle each was implemented in NS-3, as described in [1], Section VIII, Subsection B. An explanation of the aforementioned scenario follows: Each platoon consists of vehicles $3 \mathrm{~m}$ long separated from the precedent vehicle by $1 \mathrm{~m}$. Platoons are separated from one another by $30 \mathrm{~m}$. The antennas are $1.5 \mathrm{~m}$ above the ground. The platoons' leaders broadcast messages to their followers at $3 \mathrm{Mb} / \mathrm{s}$ data rate and in LOS, using a binary phase-shift keying (BPSK) orthogonal frequency division multiplexing (OFDM) modulation mode with a forward error correction (FEC) coding rate of $1 / 2$ [54]. A bit error rate (BER) versus signal-to-noise ratio (SNR) comparison study of different DSRC modulation schemes suggests that BPSK with $1 / 2$ rate code is one of the most robust [55]. Hence, transmissions to the eighth follower, which is $28 \mathrm{~m}$ apart, are more reliable, as well as emergency transmissions to neighbor platoons. Nevertheless, quadrature phase-shift keying (QPSK), working at $6 \mathrm{Mb} / \mathrm{s}$ data rate with a FEC coding rate of $1 / 2$, is also very robust, particularly if used with a threshold for a node to enter the reception state raised to the signal to interference plus noise ratio (SINR) level of QPSK [56]. The followers communicate to their own followers by unicast at $27 \mathrm{Mb} / \mathrm{s}$ data rate, using a 64-quadrature amplitude modulation (QAM) OFDM with a FEC coding rate of $3 / 4$. Although being more susceptible to noise than BPSK OFDM modulation, the used data rate is more favorable concerning the tight timings of the followers' time slots. Moreover, since followers communicate at a distance of $4 m$, and the potential contending vehicles in the same time slot are $61 \mathrm{~m}$ apart both upstream (behind) and downstream (upfront), the SNR is mostly favorable. Additionally, power control techniques and directional antennas may further improve follower-to-follower transmission reliability. Each data packet payload has $100 \mathrm{~B}$ and explicit acknowledgement (ACK) packets have $10 \mathrm{~B}$. Communications are performed through DSRC's control channel $(\mathrm{CCH})$. At the beginning of each $\mathrm{CCH}$, a $4 \mathrm{~ms}$ guard interval (GI) was defined, to allow proper synchronization and channel switching of all vehicles [54]. $11 \mathrm{~ms}$ of the remaining $46 \mathrm{~ms}$ are reserved for the leader, and each one of the seven followers uses $5 \mathrm{~ms}$ slots. Fig. 1 shows the logical division of the $\mathrm{CCH}$.

\section{B. NS-3 Simulation Results of a Single Lane Platooning}

Several simulations were performed, as described in [1]. $\mathrm{CCH}$ was divided in time slots to avoid intraplatoon collisions. Nevertheless, at the beginning of each time slot 


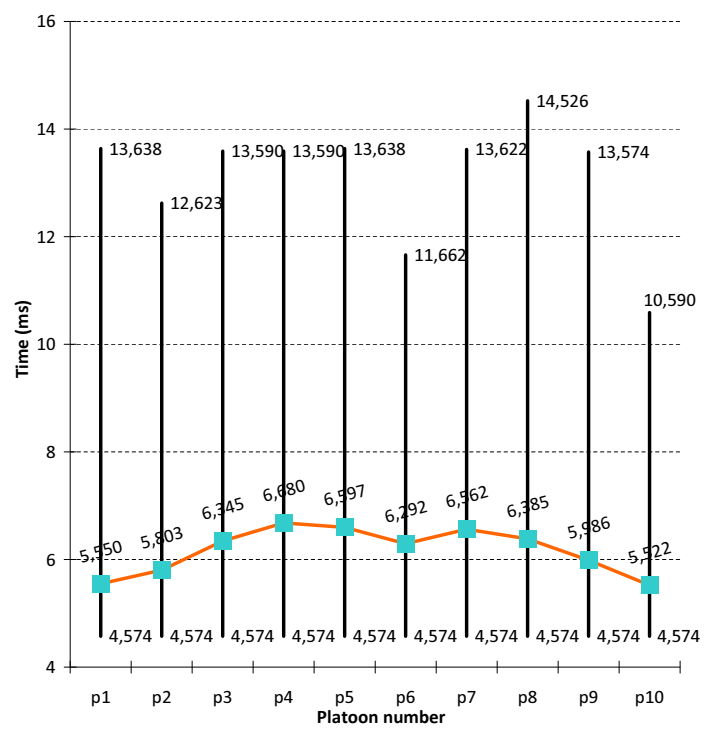

Fig. 2. Platoons' followers reception delays of messages broadcasted by their leaders, for the single lane platooning scenario (from [1]).

first packet collisions could occur, due to the interplatoons transmission synchronism. To decrease the probability of these early collisions, an additional application-level random backoff between $C W_{\min }$ and $C W_{\max }$ was defined.

Reception delays from precedent vehicles, per platoon, using unicast, were very favorable [1]. The average values were all under $1 \mathrm{~ms}$, and no failures occurred over the entire simulation. However, the broadcasted communication between the leaders and their followers raised concerns about its resilience, since it presents no packet delivery guaranty, due to the absence of ACK mechanism in broadcasting. Therefore, a broadcast emitter has no means of detecting a collision, since the MAC of IEEE 802.11p uses carrier sense multiple access with collision avoidance (CSMA/CA). This was the main reason why an explicit ACK mechanism was implemented.

The data shown in Figs. 2 and 4 were obtained from statistically non-correlated simulations. Fig. 2 presents the aggregate results of the simulations performed, with the average, minimum and maximum values of reception delays of the followers, from leaders' broadcasted messages, per platoon position. Since GI imposes a constant time penalty from the beginning of each $\mathrm{CCH}$, GI is included, along with the actual delays, as part of the total delays. Thus, the delays perceived by the followers from the beginning of each $\mathrm{CCH}$ are more clearly shown. Notwithstanding the foregoing, the successful transmission results of DSRC's broadcasted messages by the leaders are not affected, since the time slots for effective leaders' transmission are always $11 \mathrm{~ms}$ long (from $4 \mathrm{~ms}$ to $15 \mathrm{~ms}$ with the GI, or from $0 \mathrm{~ms}$ to $11 \mathrm{~ms}$ without it). The middle platoons present higher average delay values. The platoons at the front and the tail of the chain present better average delay results, whereas the platoons in the middle of the chain present slightly higher average delay results. First leaders' transmissions were mostly successful.

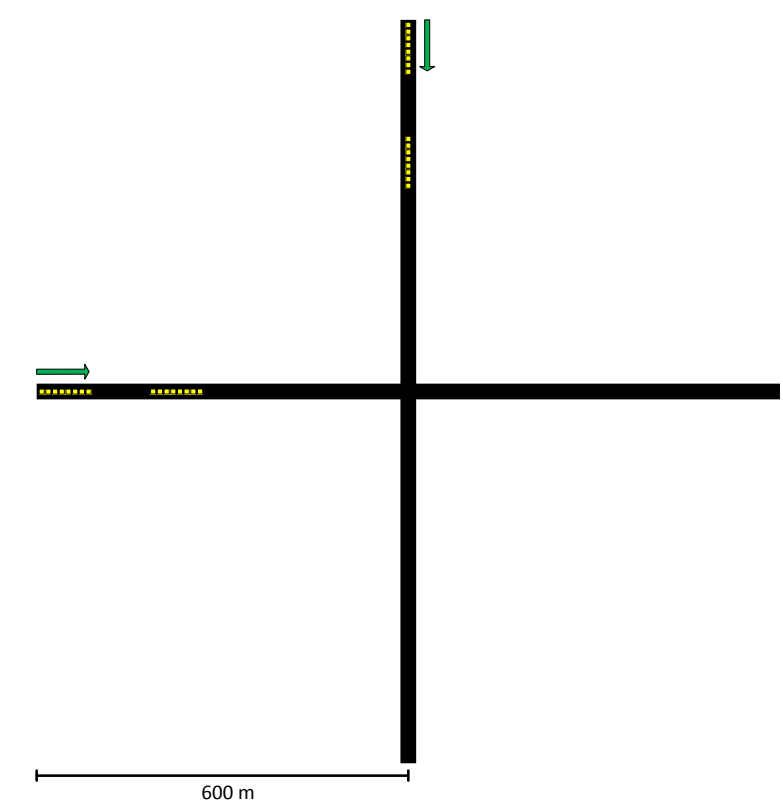

Fig. 3. Setup of the two single lanes platooning scenario, on an elevated crossroad configuration, with two perpendicular tracks $4 m$ vertically apart.

When the respective ACK failed to arrive in time, a second transmission took place. A further third transmission occurred, if the second transmission's ACK was not received in time by the leader.

\section{NS-3 Simulation Setup of Two Single Lanes Platooning}

A new scenario of two sets of ten platoons of eightvehicle each was implemented in NS-3, in a two single lane platooning, as shown in Fig. 3. Each set of ten platoons presented an analogous configuration of the platoons defined in subsection V-A. The worst case scenario with respect to communications was adopted, i.e., when the platoons "meet" they are synchronized in a way that the respective leaders pass exactly one over the other at the cross point. Therefore, leaders' broadcasting is subject to strong mutual interference.

\section{NS-3 Simulation Results of Two Single Lanes Platooning}

Reception delays from precedent vehicles, per platoon, using unicast, were almost as favorable as before. The average values were all around $1 \mathrm{~ms}$, and no failures occurred over the entire simulation.

However, the broadcasted communication between the leaders and their followers did not behave so well. In fact, a few failures occurred. Fig. 4 presents the aggregate results of the simulations performed, with the average, minimum and maximum values of reception delays of the followers, from leaders' broadcasted messages, per platoon position. In this case, higher average delay values are presented by the platoons at the front, since they suffer from stronger interference of the platoons evolving on the perpendicular track. Moreover, we can derive from the maximum delay values of three platoons (15 ms: $4 \mathrm{~ms}$ of the GI plus $11 \mathrm{~ms}$ of leader's time slot) that some collisions occurred: on the first platoon (2 nonconsecutive collisions), on the third platoon 


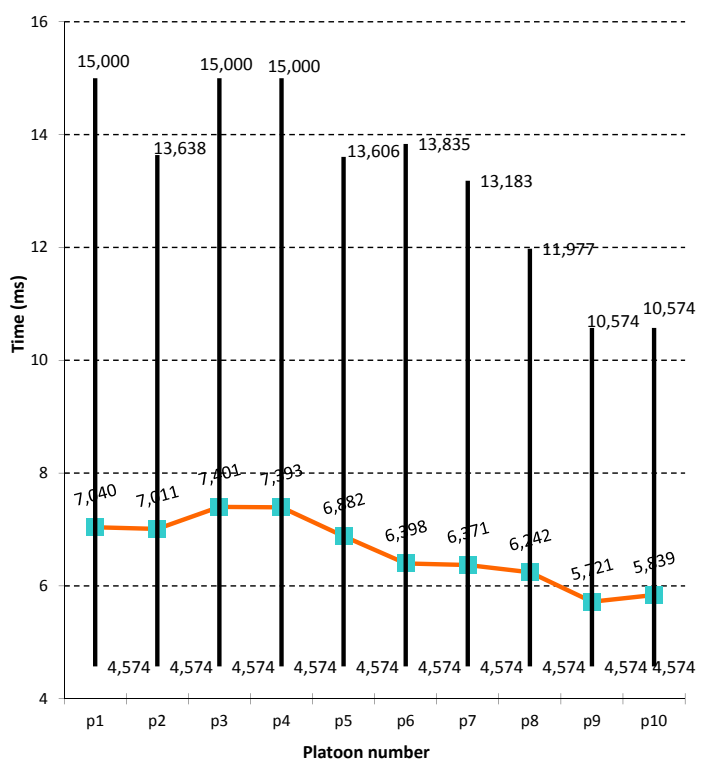

Fig. 4. Platoons' followers reception delays of messages broadcasted by their leaders, for the two single lanes platooning scenario.

(4 nonconsecutive collisions), and on the fourth platoon (3 nonconsecutive collisions). As such, the platoons' leaders present some transmission failures that may endanger platoon stability and safety. Therefore, in addition to the already aforementioned channel overload, this transmission scheme using broadcast on a relatively short time slot is not reliable enough to be adopted by a platooning system, even when using the implemented retransmission scheme. Clearly, the leaders need more time to reliably transmit their data.

\section{USING IR AND DSRC FOR COMMUNICATION RELIABILITY IMPROVEMENT}

The proposed solution to address the aforementioned DSRC problems, to timely cope with the data demand from a constant spacing platooning of autonomous vehicles, is to use IR communications for transmitting all periodic control data between platoon vehicles. This approach aims to decrease DSRC channel load and free it up from time constrained data. This way, DSRC may be able to deal with less time stringent messages, such as plans of action for the next seconds received from ATMS upper levels, or requests from other vehicles for adhesion or exit the platoon. The proposed communication scheme is shown in Fig 5. The correspondent information management framework is described as follows:

- the adoption of a transmission scheme with two different IVC technologies, i.e., DSRC and IR. This option aims to free up DSRC channel from transmitting all the periodic messages needed to control each and every follower vehicle in the platoon. Although DSRC can cope with single lane platoons, simulations suggest that more complex track networks would eventually cause DSRC to fail some data frames transmission, when working in broadcast. And a platooning system can not afford such communication unreliability, since safety would then be compromised;

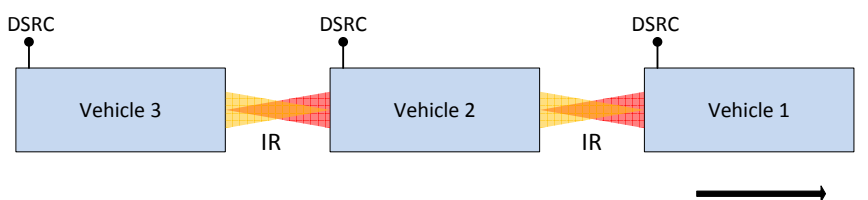

Fig. 5. IVC configuration of a platoon of vehicles, using DSRC and IR.

- the use of broadcast on the transmission through DSRC of event-driven messages, such as vehicles' request to join or leave a platoon, safety warnings, and other less time stringent messages;

- the use of unicast and the information updating scheme IV from [1] on the transmission through IR of all periodic messages within a formed platoon, such as vehicle control-based messages;

- the use of each $50 \mathrm{~ms}$-long DSRC's CCH in all its extension for messages broadcasted and received by the leaders;

- the addition of a feature toward the repetition of leaders' data through IR using unicast, which improves the communication layer proposed in [1], ensuring redundancy of leaders' broadcasted parameters to all followers through DSRC, and so increasing safety.

The development of the proposed framework at the communication level will take into account the guidelines of appropriate IEEE, ETSI, and ISO standards, including CALM.

\section{CONCLUSION}

A new IVC communication architecture for platooning systems is proposed in this paper. Through network simulations, we identified some weaknesses on DSRC if used alone in such data demand scenarios. Then, we outlined a framework to solve IVC constraints, by adding a new communication technology to each vehicle, using IR, and transmitting most of the control data through it. This way, each vehicle is able to reliably use DSRC to fulfill their communication purposes, leaving for IR the more demanding and time stringent data.

Future work will address a simulation platform that may reliably reproduce the transmission and reception conditions of IR systems when used in close formation platoons. The use of visible light communications (VLC) [57] will also be considered, and the integration of the aforementioned communication technologies will be subject of further research.

\section{REFERENCES}

[1] P. Fernandes, and U. Nunes, "Platooning with IVC-enabled autonomous vehicles: Strategies to mitigate communication delays, improve safety and traffic flow," IEEE Trans. Intelligent Transportation Systems. vol. 13, no. 1, pp. 91-106, March 2012.

[2] J. E. Anderson, "An intelligent transportation network system: Rationale, attributes, status, economics, benefits, and courses of study for engineers and planners," PRT International, 2009.

[3] IEEE Standard for Local and Metropolitan Area Networks-Specific requirements Part 11: Wireless LAN Medium Access Control (MAC) and Physical Layer (PHY) Specifications Amendment 6: Wireless Access in Vehicular Environments, IEEE 802.11p-2010, Jul. 2010.

[4] IEEE Standard for Wireless Access in Vehicular Environments (WAVE)-Networking Services, IEEE 1609.3/D8.0-2010, Jun. 2010. 
[5] IEEE Standard for Wireless Access in Vehicular Environments (WAVE)-Multi-channel Operation, IEEE 1609.4-2010, Dec. 2010.

[6] V. Milanés, J. Alonso, L. Bouraoui, and J. Ploeg, "Cooperative maneuvering in close environements among cybercars and dual-mode cars," IEEE Trans. Intelligent Transportation Systems, vol. 12, no. 1, March 2011.

[7] L. Xiao, and F. Gao, "Practical string stability of platoon of adaptive cruise control vehicles," IEEE Trans. Intelligent Transportation Systems, vol. 12, no.4, December 2011, pp. 1184-1194.

[8] B. van Arem, C. van Driel, and R. Visser, "The impact of cooperative adaptive cruise control on traffic-flow characteristics" IEEE Trans. Intelligent Transportation Systems, vol. 7, no. 4, December 2006.

[9] A. Kesting, M. Treiber, and D. Helbing, "Connectivity statistics of store-and-forward intervehicle communication " IEEE Trans. Intelligent Transportation Systems, vol. 11, no. 1, March 2010.

[10] C.-L. Huang, Y. Fallah, R. Sengupta, and H. Krishnan, "Intervehicle transmission rate control for cooperative active safety system," IEEE Trans. Intelligent Transportation Systems, vol. 12, no. 3, Sep. 2011.

[11] Y. Wang, M. Papageorgiou, J. Gaffney, I. Papamichail, and J. Guo, "Local ramp metering in the presence of random-location bottlenecks downstream of a metered on-ramp", in 2010 13th Int. IEEE Conference on Intelligent Transportation Systems, Madeira Island, Portugal, September 19-22, 2010.

[12] V. Milanés, J. Godoy, J. Villagra, and J. Perez, "Automated onramp merging system for congested traffic situations," IEEE Trans. Intelligent Transportation Systems, vol. 12, no. 2, pp. 500-508, June 2011.

[13] LTE-Advanced (Long Term Evolution). [Online]. Available: http://www.3gpp.org/LTE-Advanced

[14] P. Fernandes, and U. Nunes, "Vehicle communications: A short survey," IADIS Telecommunications, Networks and Systems, Lisbon, 2007.

[15] H. Hartenstein, and K. Laberteaux, "A tutorial on vehicular ad-hoc networks," IEEE Communications Magazine, vol.46, no. 6, pp. 164171, June 2008.

[16] M. Mahmod, I. Khalil, E. Uhlemann, and N. Nygren, "Wireless strategies for future and emerging ITS applications", 2008.

[17] Intelligent transport systems-Communications access for land mobiles (CALM)-Architecture, ISO 21217:2010(E). Apr. 2010.

[18] Intelligent transport systems-Communications access for land mobiles (CALM)-Medium service access points, ISO 21218:2008(E). Aug. 2008.

[19] B. Williams, "The CALM handbook, continuous air-interface long and medium range". ISO TC204: ETSI ERM TG37. U. K., 2004.

[20] Intelligent transport systems-Communications access for land mobiles (CALM)-M5, ISO 21215:2010(E). Nov. 2010.

[21] Intelligent transport systems-Wireless Comms.-CALM using millimetre communications-Air interface, ISO 21216:2011(E). Jan. 2011.

[22] Intelligent transport systems-Continuous air interface, long and medium range (CALM)-Infra-red systems, ISO 21214:2006(E). Jul. 2006.

[23] International Organization for Standardization (ISO). [Online]. Available: http://www.iso.org

[24] European Telecommunications Standards Institute (ETSI). [Online]. Available: http://www.etsi.org

[25] Joint standard development for Cooperative Intelligent Transport Systems (C-ITS). [Online]. Available: http://its-standards.eu

[26] Toll Collect, Service on the Road. [Online]. Available: http://www.tollcollect.de

[27] Vehicle Information and Communication Systems (VICS). [Online]. Available: http://www.vics.or.jp/english/vics/index.html

[28] NS-3, network simulator. [Online]. Available: http://www.nsnam.org

[29] T. Willke, P. Tientrakool, and N. Maxemchuk, "A survey of inter-vehicle communication protocols and their applications," IEEE Communications Surveys \& Tutorials, vol 12, no. 2, pp. 3-20, 2009.

[30] K. Dar, M. Bakhouya, J. Gaber, and M. Wack, "Wireless communication technologies for ITS applications," IEEE Communications Magazine, vol. 48, no. 5, pp. 156-162, May 2010.

[31] H. Boeglen, B. Hilt, and P. Lorentz, "A survey of V2V channel modeling for VANET simulations," $8^{\text {th }}$ Int. Conf. on Wireless OnDemand Network Systems and Services (WONS2011), Italy, 2011.

[32] F. Yu, and S. Biswas, "Self-configuring TDMA protocols for enhancing vehicle safety with DSRC based vehicle-to-vehicle communications," IEEE J. on Selected Areas in Communications, vol. 25, no. 8, Oct 2007.
[33] M. Torrent-Moreno, J. Mittag, P. Santi, and H. Hartenstein, "Vehicleto-vehicle communication: Fair transmit power control for safetycritical information," IEEE Trans. Vehicular Tecnology. vol. 58, no. 7 Sep 2009.

[34] Y. Bi, L. Cai, X. Shen, and H. Zhao, "Efficient and reliable broadcast in intervehicle communication networks: A cross-layer approach," IEEE Trans. Vehicular Technology, vol. 59, no. 5, June 2010.

[35] C. E. Palazzi, M. Rosseti, and S. Ferreti "An intervehicular communication architecture for safety and entertainment," IEEE Trans. on Intelligent Transportation Systems, vol. 11, no. 1, pp. 90-99, March 2010.

[36] S. Tabatabaei, M. Fleury, N. Qadri, and M. Ghanbari, "Improving propagation modeling in urban environments for vehicular ad hoc networks," IEEE Trans. on Intelligent Transportation Systems, vol. 12 no. 3, pp. 705-716, June 2011.

[37] M. Piorkowski, M. Raya, A. Lugo, P. Papadimitratos, M. Grossglauser, and J-P. Hubaux, "TraNS: Realistic joint traffic and network simulator for VANETs", ACM SIGMOBILE Mobile Computing and Communications Review, vol. 12, no. 1, pp. 31-33, Jan. 2008.

[38] D. Krajzewicz, M. Bonert, and P. Wagner, "The open source traffic simulation package SUMO," RoboCup 2006 Infrastructure Simulation Competition, RoboCup 2006, Germany, Bremen, 2006.

[39] NS-2 network simulator. [Online]. Available: www.isi.edu/nsnam/ns

[40] A. Wegener, M. Piorkowski, M. Raya, H. Hellbrück, S. Fischer, and J.P. Hubaux, "TraCI: An interface for coupling road traffic and network simulators," Proc. of $11^{\text {th }}$ Communications and Networking Simulation Symposium CNS'08, 2008.

[41] Hermes network simulator. [Online]. Available: http://students.ceid.upatras.gr/ xithalis/simulation_en.htm

[42] Innovative transportation simulator (iTS). [Online]. Available: http://www.maitint.org

[43] P. Fernandes, and U. Nunes, "Platooning of autonomous vehicles with intervehicle communications in SUMO traffic simulator," in 2010 13th Int. IEEE Conference on Intelligent Transportation Systems, Madeira Island, Portugal, September 19-22, 2010.

[44] S. Mahal, "Effects on communication delays on string stability in a AHS environment," M.S. Thesis, UC Berkeley, March 2000.

[45] P. Fernandes, and U. Nunes, "Algorithms for management of a multi-platooning system of IVC-enabled autonomous vehicles, with high traffic capacity," 2011 14th Int. IEEE Conference on Intelligent Transportation Systems, Washington DC, USA, October 5-7, 2011.

[46] P. Fernandes, and U. Nunes, "Platooning with IVC-enabled autonomous vehicles: Inter- and intraplatoon positioning management and cooperative behavior algorithms for high traffic capacity." Submitted for publication.

[47] P. Varaiya, "Smart cars on smart roads: Problems of control," Trans. Automatic Control, AC-38(2), pp. 195-207, 1993.

[48] M. Mio, A. Tachibana, K. Aoki, and M. Nishida, "Platoon system based on optical inter-vehicle communication," Intelligent Transport Systems World Congress, pp. 1272-1277, 1995.

[49] H. Fujii, O. Hayashi, and N. Nakagata, "Experimental research on intervehicle communication using infrared rays," Proc. IEEE Intelligent Vehicles Symp., pp. 266271, 1996.

[50] S. Tsugawa, "Issues and recent trends in vehicle safety communication systems," IATSS Research, vol. 29, no. 1, 2005.

[51] CO-OPerative SystEms for Intelligent Road Safety (COOPERS). [Online]. Available: http://www.coopers-ip.eu

[52] Cooperative Vehicle-Infrastructure Systems (CVIS). [Online]. Available: http://www.cvisproject.org

[53] A. Schalk, and H. Stratil, "The new infrared roadside transmitter brings ISO CALM on the street," Efkon AG, Austria, 2009.

[54] H. Hartenstein, and Kenneth Laberteaux, VANET Vehicular Applications and Inter-Networking Technologies, John Wiley and Sons, 2010.

[55] A. Sassi, F. Charfi, L. Kamoun, Y. Elhillali, and A. Rivenk, "OFDM transmission performance evaluation in V2X Communication", IJCSI Int. Journal of Computer Science Issues, vol. 9, is. 2, no. 3, Mar. 2012.

[56] Q. Chen, D. Jiang, V. Taliwal, and L. Desgrossi, "IEEE 802.11 based vehicular communication simulation design for NS-2", Proc. of the 3rd international workshop on Vehicular ad hoc networks, pp. 50-56, Los Angeles, U. S. A. 2006.

[57] IEEE Standard for Local and Metropolitan Area Networks - Part 15.7: Short-Range Wireless Optical Communication Using Visible Light, IEEE Std 802.15.7-2011, Sep. 2011. 